\title{
Thoracic Radiography Characteristics of Drug Sensitive Tuberculosis and Multi Drug Resistant Tuberculosis: a Study of Indonesian National Tuberculosis Prevalence Survey
}

\author{
Rosalia Sri Sulistijawati ${ }^{1}$, Aziza Ghanie Icksan², Dina Bisara Lolong³, Fariz Nurwidya4,*
}

\begin{abstract}
Background: Tuberculosis (TB) remains a burden globally, including Indonesia. The primary objective of this study is to reveal the chest radiography characteristic of drug-sensitive TB (DS-TB) and multi-drug resistant TB (MDR-TB) in the Indonesian national tuberculosis prevalence survey 2013-2014. The secondary objective is to explore the correlation and incidence rate of chest radiography lesion of DS-TB and MDR-TB cases.

Methods: This is a cross-sectional retrospective analytical studies with national and regional coverage. Samples were selected by stratified multi-stage clustering sampling technique in a population aged $\geq 15$ years old. The diagnosis of TB was based on culture and GeneXpert tests.

Results: There were 147 DS-TB and 11 MDR-TB patients that were analyzed in this study. The nodule is the only type of lesions that distinguish MDR-TB and DS-TB. In multivariate analysis of DS-TB, there were 3 significant chest radiography lesions, i.e infiltrate, cavity and consolidation with odd-ratio (OR) of 14, 13, and 3, respectively. In MDR-TB, the only significant lesion is a nodule, with OR of 19. Conclusion: Nodule is the only type of lesions that distinguish MDR-TB and DS-TB. Infiltrate, cavity and consolidation were the types of chest radiography lesions on DS-TB, meanwhile, a nodule was the only significant lesion for MDR-TB.
\end{abstract}

\section{KEYWORDS}

chest radiography; tuberculosis; DS-TB; MDR-TB

AUTHOR AFFILIATIONS

${ }^{1}$ Department of Radiology, Temanggung Regional Hospital, Central Java, Indonesia

2 Department of Radiology, Persahabatan Hospital, Jakarta, Indonesia

${ }^{3}$ National Institute of Health Research and Development, Indonesian Ministry of Health, Jakarta, Indonesia

${ }^{4}$ Department of Pulmonology and Respiratory Medicine, Faculty of Medicine Universitas Indonesia, Persahabatan Hospital, Jakarta, Indonesia

* Corresponding author: Department of Pulmonology and Respiratory Medicine, Faculty of Medicine Universitas Indonesia, Persahabatan Hospital, Jalan Persahabatan Raya No. 1, Rawamangun Jakarta 13230, Indonesia; e-mail: fariz.nurwidya@gmail.com

Received: 23 April 2018

Accepted: 22 January 2019

Published online: 1 April 2019

Acta Medica (Hradec Králové) 2019; 62(1): 24-29

https://doi.org/10.14712/18059694.2019.42

(c) 2019 The Authors. This is an open-access article distributed under the terms of the Creative Commons Attribution License (http://creativecommons.org/licenses/by/4.0), which permits unrestricted use, distribution, and reproduction in any medium, provided the original author and source are credited. 
List of abbreviations: AFB, acid-fast bacilli; CI, confidence interval; DS-TB, drug-sensitive tuberculosis; GGO, ground glass opacity; HIV, human immunodeficiency virus; MDR-TB, multidrug resistant tuberculosis; MTB, $M y$ cobacterium tuberculosis; NTM, Non-Tuberculous Mycobacterium; OR, odds ratio; TB, tuberculosis; WHO, World Health Organization; XDR-TB, extremely drug resistant tuberculosis.

\section{INTRODUCTION}

World Health Organization (WHO) predicted an increasing multi-drug resistant tuberculosis (MDR-TB) morbidity and mortality $(1,2,3)$. Diagnosis of TB by using sputum culture with MDR-TB susceptibility test takes a long time, therefore WHO developed the GeneXpert method which only takes 2 to 4 hours to diagnose MDR-TB (4). The sensitivity and specificity of GeneXpert and acid-fast bacilli (AFB) were $95.3 \%$ vs $60.5 \%$ and $86.4 \%$ vs $98.5 \%$, respectively (5).

In addition, chest radiography is the only simple, cost-effective, selected, safe, and important imaging modality to detect lesions morphology in the lung especially in pre-clinical stages. In observing TB treatment improvement, a chest $\mathrm{x}$-ray is usually performed at 2 months and 6 months after starting therapy. In TB with the negativity of AFB smear or sputum culture, diagnosis and observation of treatment improvement are based on clinical symptoms and chest radiography findings (6).

There has been no study or data described the comparison of characteristic of drug-sensitive TB (DS-TB) and MDR-TB lesions on chest radiograph in the out-hospital setting. Based on these facts, the problems of this study can be formulated, whether there is a different characteristic of DS-TB lesions of chest radiography compared with AFB smear or sputum culture and GeneXpert-based MDR$\mathrm{TB}$ on the patients of 15 years old or more in order to early detection efforts.

\section{MATERIALS AND METHODS}

This is a cross-sectional retrospective study with national and regional coverage. This study was conducted at the National Institute of Health Research and Development, Indonesian Ministry of Health, Jakarta. Data were collected for 3 consecutive days on 16-18 December 2016. Database of national TB prevalence survey 2013-2014 was used. Stratified multi-stage sampling technique was used to populations of 15 years old and above.

Subjects of the study were collected from the secondary data at the Ministry of Health., the Republic of Indonesia. The sample of this study were patients of 15 years old or over with a complaint of coughing of more than 14 days or coughing up blood, who performed chest radiograph study, sputum AFB smear and continued with culture for Mycobacterium tuberculosis (MTB) and GeneXpert test, lived in special cluster area at least for one month, excluding individuals living in military barracks, diplomatic mission homes, hospitals, hotels, dormitories, and temporary shelters. The subjects should fulfil the inclusion criteria of patients with MTB chest radiograph, with a final diagnosis of MDR-TB and with the final barcode diagnosis DS-TB.

The exclusion criteria are patients with incomplete medical record data, with chest radiograph indicating other abnormalities of parenchyma, with massive pleural effusion and pneumothorax causing the lung parenchyma could not be assessed. Direct Digital Radiography DICOM (Agva Healthcare, South Carolina, US) was used to perform chest X-ray and the results were read by 3 thoracic radiologists.

The independent variables studied were locations of the lesion on the chest radiograph, whether on the right or left lung. The upper field is an area restricted to lung apex, 2nd anterior costae, and midline projected from procesus spinosus. The medium field is an area restricted from the 2nd to 4 th costae and midline projected from processus spinosus. The lower field in an area restricted from the 4th to 6 th costae and midline projected from processus spinosus. The type of lesions studied as independent variables were infiltrate, cavity, nodule, mass, consolidation, pleural effusion, pleural thickening (thickening of the pleura), fibrosis and calcification. The dependent variables studied were MDR-TB and DS-TB. MDR-TB is defined as sputum AFB (+/-), culture (+) and GeneXpert (+), meanwhile DSTB is defined as sputum AFB (+/-), culture (+) and GeneXpert (-). Data collection is done by reviewing the medical record of each patient. The data were analyzed using the Statistical Package for Social Science (SPSS) software program version 21 (IBM Corp, Armonk, NY, USA). Chi-square $\left(\mathrm{x}^{2}\right)$ data processing was used for bivariate analysis and logistic regression for multivariate analysis. $P<0.05$ was considered to be statistically significant.

\section{RESULTS}

Participants who met inclusion and exclusion criteria were 147 DS-TB and 11 MDR-TB patients (Tabel 1). Non-Tuberculous Mycobacterium (NTM) patients were included

Tab. 1 Distribution of participant according to characteristics.

\begin{tabular}{|l|l|l|}
\hline Characteristic & DS-TB & MDR-TB \\
\hline Age group & 47 & 6 \\
\hline $15-34$ & 58 & 4 \\
\hline $35-54$ & 42 & 1 \\
\hline$>55$ & \multicolumn{2}{|l|}{} \\
\hline Sex & 107 & 9 \\
\hline Man & 40 & 2 \\
\hline Woman & \multicolumn{2}{|l|}{} \\
\hline History of tuberculosis treatment & 112 & 3 \\
\hline No & 35 & 8 \\
\hline Yes & \multicolumn{2}{|l|}{} \\
\hline
\end{tabular}

Abbreviations: DS-TB, drug sensitive tuberculosis; MDR-TB, multidrug resistant tuberculosis. 
Tab. 2 Association of lesion on chest radiograph and DS-TB.

\begin{tabular}{|c|c|c|c|c|c|c|c|c|c|}
\hline \multirow{2}{*}{\multicolumn{2}{|c|}{ Variables }} & \multicolumn{4}{|c|}{ DS-TB } & \multirow{3}{*}{$\mathbf{P}$} & \multirow{3}{*}{ OR } & \multirow[b]{3}{*}{ Lower } & \multirow[b]{3}{*}{ Upper } \\
\hline & & \multicolumn{2}{|l|}{ Yes } & \multicolumn{2}{|l|}{ No } & & & & \\
\hline & & $\mathbf{F}$ & $\%$ & $\mathbf{F}$ & $\%$ & & & & \\
\hline \multirow{2}{*}{ Infiltrate } & Present & 96 & 67.6 & 46 & 32.4 & \multirow{2}{*}{0.000} & \multirow{2}{*}{13.708} & \multirow{2}{*}{8.666} & \multirow{2}{*}{21.685} \\
\hline & No & 51 & 13.2 & 335 & 86.8 & & & & \\
\hline \multirow{2}{*}{ Cavity } & Present & 41 & 73.2 & 15 & 26.8 & \multirow{2}{*}{0.000} & \multirow{2}{*}{9.438} & \multirow{2}{*}{5.028} & \multirow{2}{*}{17.715} \\
\hline & No & 106 & 22.5 & 366 & 77.5 & & & & \\
\hline \multirow{2}{*}{ Nodule $<3 \mathrm{~cm}$} & Present & 28 & 60.9 & 18 & 39.1 & \multirow{2}{*}{0.000} & \multirow{2}{*}{4.745} & \multirow{2}{*}{2.534} & \multirow{2}{*}{8.885} \\
\hline & No & 119 & 24.7 & 363 & 75.3 & & & & \\
\hline \multirow{2}{*}{ Mass } & Present & 1 & 11.1 & 8 & 88.9 & \multirow{2}{*}{0.249} & \multirow{2}{*}{0.319} & \multirow{2}{*}{0.040} & \multirow{2}{*}{2.576} \\
\hline & No & 146 & 28.1 & 373 & 71.9 & & & & \\
\hline \multirow{2}{*}{ Consolidation } & Present & 55 & 72.4 & 21 & 27.6 & \multirow{2}{*}{0.000} & 10210 & & \\
\hline & No & 92 & 20.4 & 360 & 79.6 & & 10.240 & ס & 17.001 \\
\hline Ploural ffucion & Present & 17 & 68.0 & 8 & 32.0 & 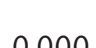 & 6097 & 2571 & 14162 \\
\hline काent at entusiont & No & 130 & 25.8 & 373 & 74.2 & 0.000 & (0.0 & $2.5 / 1$ & 14.402 \\
\hline Ploural thickening & Present & 13 & 59.1 & 9 & 40.9 & 0001 & 1010 & 1676 & 0596 \\
\hline Се & No & 134 & 26.5 & 372 & 73.5 & 0.001 & 4.010 & 1.070 & (2) \\
\hline Fihrocic & Present & 45 & 50.0 & 45 & 50.0 & 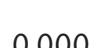 & 3294 & 2061 & 5265 \\
\hline (10) & No & 102 & 23.3 & 336 & 76.7 & 0.000 & 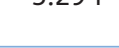 & 2.001 & 5.203 \\
\hline Colrification & Present & 14 & 41.2 & 20 & 58.8 & 0072 & 1900 & 0022 & 2070 \\
\hline Cateinicationit & No & 133 & 26.9 & 361 & 73.1 & S & 1.500 & נכבכ. & 5.070 \\
\hline
\end{tabular}

Abbreviations: DS-TB, drug-sensitive tuberculosis; OR, odd-ratio.

in statistical data calculation, aiming to avoid any bias in the description of lesions characteristic. First, we determined the association of lesion on chest radiograph and DS-TB incidence. As seen in Table 2, infiltrate, cavity, nodule, consolidation, pleural effusion, pleural thickening, and fibrosis were significantly associated with DS-TB incidence.

We then clarified what lesion is predominantly found in DS-TB by performing multivariate analysis (logistic

Tab. 3 Result of Multivariate analysis of lesions on Chest radiography DS-TB.

\begin{tabular}{|l|l|l|l|l|}
\hline \multirow{2}{*}{ Variables } & \multirow{2}{*}{ Sig. } & \multirow{2}{*}{ OR } & \multicolumn{2}{|l|}{$\mathbf{9 5 . 0 \%}$ CI for EXP(B) } \\
\cline { 5 - 6 } & & & Lower & Upper \\
\hline Infiltrate & 0.000 & 13.706 & 7.833 & 23.982 \\
\hline Cavity & 0.006 & 2.949 & 1.367 & 6.364 \\
\hline Nodul & 0.375 & 0.629 & 0.226 & 1.751 \\
\hline Mass & 0.839 & 0.788 & 0.079 & 7.844 \\
\hline Consolidation & 0.000 & 12.767 & 5.661 & 28.796 \\
\hline Pleural effusion & 0.150 & 2.157 & 0.757 & 6.143 \\
\hline Pleural thickening & 0.717 & 0.814 & 0.267 & 2.478 \\
\hline Fibrosis & 0.975 & 0.989 & 0.510 & 1.919 \\
\hline Calcification & 0.563 & 1.359 & 0.480 & 3.852 \\
\hline
\end{tabular}

Abbreviations: DS-TB, drug-sensitive tuberculosis; $\mathrm{Cl}$, confidence interval; OR, odd-ratio. regression). Based on findings in Table 3 , there were three types of lesions (infiltrate, cavity, and consolidation) having a $P$ value of $<0.05$. Thus it is concluded that infiltrate, cavities and consolidations features can be used to predict the presence of DS-TB.

Next, we analyze the association of lesion on chest radiograph and MDR-TB incidence. Based on the findings in Table 4, infiltrate, cavity, nodule, and fibrosis were significantly associated with MDR-TB incidence. We also performed multivariate analysis of lesions on chest radiograph in MDR-TB. We found that nodule has P value of $<0.05$, which mean that nodule can be used to predict the presence of MDR-TB (Table 5).

Finally, we compared the chest radiography characteristics of DS-TB and MDR-TB. A different test is done by chi squre $\left(\mathrm{x}^{2}\right)$ test. Based on the analysis in Table 6 , nodular lesions have $P$ value of $<0.05$, which suggest that nodule is the only type of lesion that can distinguish MDR-TB and DS-TB.

\section{DISCUSSION}

MDR-TB is highly prevalent and studies to reveal the role of radiology imaging in the identification of patients who are suspected to have MDR-TB are under intensive investigation. The diagnosis of TB is established on the basis of clinical history, physical examination, radiology, and bacteriological study. GeneXpert test was used for rapid 
Tab. 4 Association of lesions on chest radiograph and MDR-TB.

\begin{tabular}{|c|c|c|c|c|c|c|c|c|c|}
\hline \multirow{2}{*}{\multicolumn{2}{|c|}{ Variables }} & \multicolumn{4}{|c|}{ MDR-TB } & \multirow{5}{*}{$\begin{array}{l}\mathbf{P} \\
0.010\end{array}$} & \multirow{5}{*}{$\begin{array}{l}\text { OR } \\
5.053\end{array}$} & \multirow{2}{*}{\multicolumn{2}{|c|}{ C.I $95 \%$}} \\
\hline & & \multirow{3}{*}{\begin{tabular}{|l|} 
Yes \\
F \\
7
\end{tabular}} & \multirow{3}{*}{\begin{tabular}{|l|}
$\%$ \\
5.0
\end{tabular}} & \multirow{3}{*}{\begin{tabular}{|l|} 
No \\
F \\
133
\end{tabular}} & \multirow{3}{*}{\begin{tabular}{|l|}
$\%$ \\
95.0
\end{tabular}} & & & & \\
\hline & & & & & & & & Lower & Upper \\
\hline \multirow{2}{*}{ Infiltrate } & Yes & & & & & & & 1956 & 17520 \\
\hline & No & 4 & 1.0 & 384 & 99.0 & & & 1.456 & 11.533 \\
\hline \multirow{2}{*}{ Cavity } & Yes & 4 & 7.1 & 52 & 92.9 & \multirow{2}{*}{0.021} & \multirow{2}{*}{5.110} & \multirow{2}{*}{1.447} & \multirow{2}{*}{18.04} \\
\hline & No & 7 & 1.5 & 465 & 98.5 & & & & \\
\hline \multirow{2}{*}{ Nodule $<3 \mathrm{~cm}$} & Yes & 5 & 11.1 & 40 & 88.9 & \multirow{2}{*}{0.001} & \multirow{2}{*}{9.938} & \multirow{2}{*}{2.905} & \multirow{2}{*}{33.995} \\
\hline & No & 6 & 1.2 & 477 & 98.8 & & & & \\
\hline \multirow{2}{*}{ Mass } & Yes & 0 & 0.0 & 9 & 100.0 & \multirow{2}{*}{1.000} & \multirow{2}{*}{ - } & \multirow{2}{*}{ - } & \multirow{2}{*}{ - } \\
\hline & No & 11 & 2.1 & 508 & 97.9 & & & & \\
\hline \multirow{2}{*}{ Consolidation } & Yes & 4 & 5.3 & 71 & 94.7 & \multirow{2}{*}{0.057} & \multirow{2}{*}{3.590} & \multirow{2}{*}{1.025} & \multirow{2}{*}{12.576} \\
\hline & No & 7 & 1.5 & 446 & 98.5 & & & & \\
\hline \multirow{2}{*}{ Pleural effusion } & Yes & 1 & 4.0 & 24 & 96.0 & \multirow{2}{*}{0.417} & \multirow{2}{*}{2.054} & \multirow{2}{*}{0.253} & 16700 \\
\hline & No & 10 & 2.0 & 493 & 98.0 & & & & $16 . / 09$ \\
\hline Dlo mal thict & Yes & 2 & 9.5 & 19 & 90.5 & 007 & 5025 & 1177 & 20026 \\
\hline Pleurat tnickening & No & 9 & 1.8 & 498 & 98.2 & $0.06 /$ & $5.8<5$ & $1.1 / 7$ & $28.8<0$ \\
\hline Fibresir & Yes & 5 & 5.7 & 83 & 94.3 & 0010 & 1357 & 1300 & 14610 \\
\hline FIbrosis & No & 6 & 1.4 & 434 & 98.6 & 0.010 & $4.35 /$ & 1.300 & 14.610 \\
\hline Colcifation & Yes & 1 & 3.1 & 31 & 96.9 & 0501 & 1568 & 0101 & 12612 \\
\hline edistilicatiom & No & 10 & 2.0 & 486 & 98.0 & 0.501 & 1.500 & 0.174 & $12.04 \mathrm{~J}$ \\
\hline
\end{tabular}

Abbreviations: $\mathrm{F}$, frequency; MDR-TB, multidrug resistant tuberculosis; $\mathrm{Cl}$, confidence interval; $\mathrm{OR}$, odd-ratio.

diagnosis of MDR-TB and it is mandatory to perform the drug susceptibility testing of isoniazid and rifampicin. Chest radiography is a simple, cost-effective, selective, safe and important imaging modality in detecting morphology of lung lesions, especially in pre-clinical stage. In addition, the extent of lesions, activity of lesions, pleural involvement and complications such as fungal infection and bronchiectasis can be assessed by using chest radiography.

Tab. 5 Multivariate analysis of lesions on chest radiograph in MDR-TB.

\begin{tabular}{|l|l|c|l|r|}
\hline \multirow{2}{*}{ Variables } & \multirow{2}{*}{ Sig. } & \multirow{2}{*}{ OR } & \multicolumn{3}{|l|}{$\mathbf{9 5 . 0 \%}$ CI for EXP(B) } \\
\cline { 4 - 6 } & & & Lower & Upper \\
\hline Infiltrate & 0.281 & 2.147 & 0.536 & 8.606 \\
\hline Cavity & 0.232 & 2.428 & 0.566 & 10.410 \\
\hline Nodule & 0.003 & 18.812 & 2.627 & 134.692 \\
\hline Mass & 0.999 & 0.000 & 0.000 & \\
\hline Consolidation & 0.194 & 0.255 & 0.032 & 2.006 \\
\hline Pleural effusion & 0.866 & 1.234 & 0.107 & 14.282 \\
\hline Pleural thickening & 0.435 & 2.224 & 0.299 & 16.547 \\
\hline Fibrosis & 0.149 & 2.871 & 0.686 & 12.016 \\
\hline Calsification & 0.589 & 0.524 & 0.050 & 5.483 \\
\hline
\end{tabular}

Abbreviations: MDR-TB, multidrug resistant tuberculosis; $\mathrm{Cl}$, confidence interval; OR, odd-ratio.
This study determined the association of lesions with the incidence of DS-TB and MDR-TB. Infiltrates, cavities, nodules, consolidation, pleural effusion, pleural thickening, fibrosis, and calcification are significantly associated with the incidence of DS-TB. Furthermore, the multivariate analysis revealed three lesions (infiltrates, cavities and consolidations) that can be used to predict the presence of DS-TB. In the MDR-TB, the infiltrates, cavities, nodules, and fibrosis, were associated with the incidence of MDR-TB.

According to a study conducted by Zahirifard et al., MDR-TB has more destructive lung features when compared with people with DS-TB (7). The study found that the presence of multiple cavities, nodular lesions, infiltrates and pleural effusions is a major sign of MDR-TB when compared to DS-TB.

Based on the study conducted by Yeom et al., in 2009 in South Korea on radiological features of patients with DS$\mathrm{TB}$ and MDR-TB, it was found that lobular consolidation was more common in patients with DS-TB (8). In addition, the cavity also has a fairly significant prediction value. In general, multiple cavity formations are more common in MDR-TB patients. In addition to the above characteristics, the regression analysis in this study concluded that unilateral parenchymal lesions are more common in people with DS-TB. Yeom et al., also suggested that the level of endemicity of a country and population setting can provide different radiological characteristics so that thorough 
Tab. 6 Comparison of characteristic of lesion on chest radiograph in DS-TB with MDR-TB.

\begin{tabular}{|c|c|c|c|c|c|c|c|c|c|}
\hline \multirow{3}{*}{ Variables } & & \multicolumn{4}{|c|}{ Result } & \multirow{3}{*}{$P$ value } & \multirow{3}{*}{ OR } & \multirow{2}{*}{\multicolumn{2}{|c|}{ C.I $95 \%$}} \\
\hline & & \multicolumn{2}{|c|}{ MDR-TB } & \multicolumn{2}{|c|}{ DS-TB } & & & & \\
\hline & & $\mathbf{F}$ & $\%$ & $\mathbf{F}$ & $\%$ & & & Lower & Upper \\
\hline \multirow{2}{*}{ Infiltrate } & Yes & 7 & 6.8 & 96 & 93.2 & \multirow{2}{*}{1.000} & \multirow{2}{*}{0.930} & \multirow{2}{*}{0.260} & \multirow{2}{*}{3.326} \\
\hline & No & 4 & 7.3 & 51 & 92.7 & & & & \\
\hline \multirow{2}{*}{ Cavity } & Yes & 4 & 8.9 & 41 & 91.1 & \multirow{2}{*}{0.510} & \multirow{2}{*}{1.477} & \multirow{2}{*}{0.411} & \multirow{2}{*}{5.315} \\
\hline & No & 7 & 6.2 & 106 & 93.8 & & & & \\
\hline \multirow{2}{*}{ Nodule $<3 \mathrm{~cm}$} & Yes & 5 & 15.2 & 28 & 84.8 & \multirow{2}{*}{0.038} & \multirow{2}{*}{3.542} & \multirow{2}{*}{1.008} & \multirow{2}{*}{12.438} \\
\hline & No & 6 & 4.8 & 119 & 95.2 & & & & \\
\hline \multirow{2}{*}{ Mass } & Yes & 0 & 0.0 & 1 & 100.0 & \multirow{2}{*}{1.000} & & & \\
\hline & No & 11 & 7.0 & 146 & 93.0 & & & & \\
\hline \multirow{2}{*}{ Consolidation } & Yes & 4 & 6.8 & 55 & 93.2 & \multirow{2}{*}{1.000} & \multirow{2}{*}{0.956} & \multirow{2}{*}{0.268} & \multirow{2}{*}{3.414} \\
\hline & No & 7 & 7.1 & 92 & 92.9 & & & & \\
\hline \multirow{2}{*}{ Pleural effusion } & Yes & 1 & 5.6 & 17 & 94.4 & \multirow{2}{*}{1.000} & \multirow{2}{*}{0.765} & \multirow{2}{*}{0.092} & \multirow{2}{*}{6.351} \\
\hline & No & 10 & 7.1 & 130 & 92.9 & & & & \\
\hline Ploural thickoning & Yes & 2 & 13.3 & 13 & 86.7 & 0280 & 2201 & 0147 & 11714 \\
\hline Pleurat LIICкеIIIIg & No & 9 & 6.3 & 134 & 93.7 & 0.200 & 2.291 & 0.441 & $11 . / 44$ \\
\hline Fihrocis & Yes & 5 & 10.0 & 45 & 90.0 & 307 & 1889 & 0548 & 6511 \\
\hline riviUsis & No & 6 & 5.6 & 102 & 94.4 & 0.507 & 1.000 & 0.040 & $0 . J 11$ \\
\hline Calsification & Yes & 1 & 6.7 & 14 & 93.3 & $100 \Omega$ & 0950 & 0113 & 7979 \\
\hline & No & 10 & 7.0 & 133 & 93.0 & 1.000 & 0.500 & o & הוב \\
\hline
\end{tabular}

Abbreviations: F, frequency; DS-TB, drug sensitive tuberculosis; MDR-TB, multidrug resistant tuberculosis; $\mathrm{Cl}$, confidence interval; OR, odd-ratio.

study and analysis of all TB endemic countries is needed to obtain more accurate results.

In 2011, Kim et al., conducted a study to compare CT Scan features in MDR-TB and DS-TB among human immunodeficiency virus (HIV) negative patients (9). In that univariate analysis, it found that bronchiektasis, pulmonary destruction, granuloma calcification, and cavity formation are often found in patients with MDR-TB. The result of the multivariate analysis showed that cavity formation was the only characteristic that differentiated MDR-TB and DS-TB.

We have previously described that there was no significant difference in morphology and lesion location on the chest radiograph of both pulmonary tuberculosis with negative AFB smear and positive culture and negative AFB smear and negative culture (10). Pulmonary TB with negative AFB smear and positive culture lung tend to have more extent lesions. Chest radiograph of 67 pulmonary TB patients with negative AFB smear and positive culture and 89 pulmonary TB patients with negative AFB smear and negative culture patients mostly showed infiltrate $(41.0 \%$ vs $32.0 \%$ ) followed by cavities ( $14.2 \%$ vs $12.2 \%$ ), consolidation $(12.4 \%$ vs $14.2 \%)$ and pleural effusion ( $0.33 \%$ vs $0.35 \%)$.

In this study, a different test was performed by Chisquare $\left(\mathrm{x}^{2}\right)$ test on the comparison of DS-TB and MDR-TB characteristics. Based on the analysis, nodules is the only the type of lesion that was significantly typical for DS-TB with an OR value of 3.5 compared to MDR-TB. This finding is consistent with our previous study and the study by Cha et al., who examined the radiographic features of patients with MDR-TB, XDR TB and compared it with DS-TB $(11,12)$. They found that the features of nodules and ground glass opacity (GGO) were more prevalent in TB patients who were still sensitive to anti TB drugs (DS-TB). While the radiological features in patients with MDR-TB and XDR TB are multiple cavities, nodules, and bronchial dilatation. Radiologic features of patients with MDR-TB and XDR TB were not significantly different. The study also discovered that patients with MDR-TB and XDR TB were younger than the DS-TB patients. Further study was necessary to see if there were multiple nodules and cavities and bronchial dilatation in young TB patients.

The study is limited with the fact that the MDR-TB cases were only consist of eleven cases. This may implicate in the limited conclusion to a broader population. Other limitation is that several subjects with non-tuberculosis mycobacterium (NTM) were included in the analysis together with TB patients.

\section{CONCLUSION}

As a conclusion, nodules are the only characteristic lesions in chest radiographs that distinguish between MDR-TB and DS-TB. The lesions that are significantly associated with the DS-TB were infiltrates, cavities, nodules, consolidations, pleural effusions, pleural thickening, fibrosis and calcification, and the OR of infiltrates, consolidation, 
and cavity were 14,13 and 3 , respectively. The lesions that are significantly associated with MDR-TB were infiltrates, cavities, nodules and fibrosis, and the OR of the nodule was 19 in MDR-TB.

\section{REFERENCES}

1. Word Health Organization, 2014. Global Tuberculosis Report 2014. Geneva: Word Health Organization.

2. Word Health Organization, 2009. A brief history of tuberculosis control in Indonesia. Geneva Word Health Organization

3. Word Health Organization, 2017. Global Tuberculosis Report 2017. Geneva: Word Health Organization.

4. World Health Organization, 2008. Management of MDR-TB: A field guide. Geneva: World Health Organization.

5. Pinyopornpanish K, Chaiwarith R, Pantip C, et al. Comparison of Xpert MTB/RIF Assay and the Conventional Sputum Microscopy in Detecting Mycobacterium tuberculosis in Northern Thailand. Tuberc Res Treat 2015; 2015: 571782.

6. Icksan AG, Luhur R. 2008. Thoracic Radiology of Pulmonary Tuberculosis, the First Edition [Radiologi toraks TB paru, Edisi Pertama]. Jakarta. CV Sagung Seto.
7. Zahirifard S, Amiri MV, Karam B, et al. The Radiological Spectrum of Pulmonary Multidrug-Resistant Tuberculosis In HIV-Negative Patients. Iran J Radiol 2003; 1: 161-6.

8. Yeom JA, Jeong YJ, Jeon D, et al. Imaging findings of primary multi drugresistant tuberculosis: A comparison of findings of drug-sensitive tuberculosis. J Comput Assist Tomogr 2009; 33: 956-60.

9. Kim HC, Goo JM, Park SH, Park CM, Kim TG. Multidrug-resistant TB versus Drug-sensitive TB in human immunodeficiency virus-negative patoient: computed tomgraphy features. J Comput Assist Tomogra 2004; 28: 366-71.

10. Icksan AG, Maryastuti M. Characteristics of chest X-Ray lesion in smear-negative TB patient with culture-positive vs culture-negative in the Persahabatan Hospital, Jakarta. Bul Ilmiah Radiol 2012; 2: 80-90. [Indonesian Society of Radiology chapter Yogyakarta and Department of Radiology, University of Gajah Mada Faculty of Medicine, Yogyakarta].

11. Icksan AG, Napitupulu MRS, Nawas MA, Nurwidya F. Chest X-ray Findings Comparison Between Multi-drug-resistant Tuberculosis and Drug-sensitive Tuberculosis. J Nat Sci Biol Med 2018; 9: $42-5$.

12. Cha J, Lee HY, Lee KS, et al. Radiological Findings of Extensively Drug-Resistant Pulmonary Tuberculosis in Non-AIDS Adults: Comparisons with Findings of Multidrug-Resistant and Drug-Sensitive Tuberculosis. Korean J Radiol 2009; 10: 207-16. 